an indirect assessment of the stage of renal amyloidosis, which may have a prognostic value in everyday surgical pathology.

Half of the patients with sAAa died of uremia caused by massive rAAa and only 9 of these were clinically recognized. Renal amyloid A deposition should be considered a very serious, life-threatening complication of RA.

Disclosure of Interest: None declared

DOI: 10.1136/annrheumdis-2018-eular.1289

\section{SAT0153 ANALYSIS OF CLINICAL-ANALYTICAL CHARACTERISTICS IN PATIENTS WITH RHEUMATOID ARTHRITIS (RA) AND INTERSTITIAL LUNG DISEASE (ILD): CASE-CONTROL STUDY}

N. Mena-Vázquez ${ }^{1}$, S. Manrique-Arija ${ }^{1}$, C. Romero-Barco ${ }^{2}$, I. Ureña-Garnica ${ }^{1}$, M. Ordóñez-Cañizares ${ }^{1}$, F. Jiménez-Núñez ${ }^{1}$, G. Díaz-Cordovés ${ }^{1}$, M. RojasGiménez $^{1}$, C. Fuego ${ }^{1}$, R. Caparrós-Ruiz ${ }^{1}$, L. Cano-Garcia ${ }^{1}$, A. Belmonte ${ }^{1}$, M. Irigoyen-Oyarzábal ${ }^{1}$, A. Fernández-Nebro ${ }^{3} .{ }^{1}$ UGC de Reumatología, Instituto de Investigación Biomédica de Málaga (IBIMA) Hospital Regional Universitario de Málaga, Universidad de Málaga, Málaga, Spain., ${ }^{2}$ UGC de Reumatología, Instituto de Investigación Biomédica de Málaga (IBIMA), Hospital Clínico Virgen de la Victoria, Universidad de Málaga, Málaga, Spain., ${ }^{3}$ UGC de Reumatología, Instituto de Investigación Biomédica de Málaga (IBIMA) Hospital Regional Universitario de Málaga, Departamento de Medicina y Dermatología, Universidad de Málaga., Málaga, Spain

Objectives: To study the differences in severity marker and disease activity in patients with RA and ILD and patients with RA without DILD, and to identify factors associated with ILD in RA patients.

Methods: Design:Observational case-control study.Patients: consecutive RApatients (ACR/EULAR 2010 criteria) with ILD (American Thoracic Society) selected from a prospective cohort from Regional Hospital and Virgen Victoria Hospital of Málaga were included. Controls: RA-patients without ILD. Sex-age matched controls were collected from a prospective cohort of Regional Hospital. Protocol: RA Patients are reviewed every six months in general clinic and patients with biological terapy every three months. All patients are reviewed according to a protocol with systematic data collection. The data of patients with RA and ILD are also collected in a database according to a specific protocol for these patients. The day that was reviewed the last time in consultation will be marked as inclusion date. Data will be collected on the date of inclusion and their clinical records. Outcomes: Difference in severity marker in both groups on the date of inclusion (RF, ACPA, erosive arthritis); in disease Activity Score (DAS28-ESR) and Health Assessment Questionnaire (HAQ); description of modifying antirheumatic drugs (DMARDs);Variables: Demographic, clinical-analytical variables: number of tender joints (TJ), number of swollen joints (SJ), CRP, ESR, general evaluation, DAS28-ESR, HAQ and adverse effects (description, severity and number).Statistical analysis: Descriptive and paired T-test or Chi-square test followed by binary logistic regression (RLB) (Vd:ILD in patients with RA).

\begin{tabular}{|c|c|c|c|}
\hline VARIABLE & RA. ILD (n=29) & $R A(n=24)$ & P. value \\
\hline Age (years), mean (SD) & $67.7(8.0)$ & $66.9(7,2)$ & 0.692 \\
\hline Sex (female), $n(\%)$ & $16(55.2)$ & $13(54.2)$ & 0.942 \\
\hline Sex (male), $n(\%)$ & $13(44.8)$ & $11(45.8)$ & \\
\hline Smoke & & & 0.003 \\
\hline Non-smoker, $n\left(\xi_{0}\right)$ & $13(44.8)$ & $16(66.7)$ & \\
\hline Ex-smoker, n(\%) & $11(37.9)$ & $0(0.0)$ & \\
\hline Smoker, $n(\%)$ & $5(17.2)$ & $8(33.3)$ & \\
\hline Disease duration RA median (p75-p25) & $130.9(91.4-208.2)$ & $99.9(73.4-121.8)$ & 0.002 \\
\hline Sodio Economic Status & & & 0.500 \\
\hline No income, $n(\%)$ & $9(31.0)$ & $8(3.3)$ & \\
\hline c1500 $/$ month, $n$ (\%) & $17(58.6)$ & $11(45.8)$ & \\
\hline$=1500 \mathrm{E} / \operatorname{month}, n(\%)$ & $3(10.3)$ & $s(20.8)$ & \\
\hline Educational level & & & 0.345 \\
\hline None, $n(\%)$ & $12(44,4)$ & $6(25.0)$ & \\
\hline Primary and secondary school, $n(\%)$ & $12(44.4)$ & $14(58.3)$ & \\
\hline University, $n$ (\%) & $3(11.1)$ & $4(16.7)$ & \\
\hline Rheumatoid factor, $n$ (\%) & $27(93.1)$ & $20(83.3)$ & 0.264 \\
\hline Anti-cydic cirullinated peptide, $n(\%)$ & $27(93.1)$ & $20(83.3)$ & 0.264 \\
\hline Anti-cyclic citrullinated peptide elevated $(3340), n(\%)$ & $22(75.9)$ & $10(43.5)$ & 0.008 \\
\hline Erosions, n (\%) & $24(82.8)$ & $12(50.0)$ & 0.011 \\
\hline
\end{tabular}

Results: Fifty-three patients were included, 29 RA with ILD and 24 RA controls. The differences between clinical and epidemiological characteristcs to cases and controls are shown in table 1. RA patients with ILD showed more months with RA duration $(p=0.002)$, more number of exsmokers $(p=0.003)$, erosive arthritis $(p=0.011)$ and ACPA positive $(P=0.008)$. No significant differences in the mean of
DAS28 in cases and controls were observed ( 2.61 vs $2.68 ; p=0.789$ ), but RA patients with ILD presented worse in physical function parameters by HAQ (1.12 VS 0.63; $P=0.032)$. All patients were treated with disease modifying antirheumatic drugs (DMARDs). RA patients with ILD had: 5 (17.2\%) monotherapy with bDMARDs, 17 (58.62) monotherapy with sDMARD and 7 (24.1) sDMARDs with a bDMARDs. In multivariate analysis, the independent variables that were associated with ILD in RA patients were: ACPA elevated (OR [IC95\%] =5.0 [1.2-9.9] $\mathrm{p}=0.023)$ and RA duration (months) (OR [IC95\%] $=1.1[1.0-1,2] ; p=0.037)$. This model would explain $28 \%$ of the variability of the ILD in $R A(R 2=0.28)$.

Conclusions: The evolution time of arthritis and the presence of ACPA to high titres ( $>340$ ) were the predictors of ILD in patients with RA in our study. More prospective studies with a greater number of patients are necessary to identify the possible association.

Disclosure of Interest: None declared

DOI: 10.1136/annrheumdis-2018-eular.4129

\section{SAT0154 ASSESSMENT OF BONE TURNOVER MARKERS IN PRE- AND POSTMENOPAUSAL WOMEN WITH RHEUMATOID ARTHRITIS}

O. Garmish ${ }^{1}$, T. Orlyk ${ }^{1}$, A. Romanovskyi ${ }^{1}$, T. Gavrylenko ${ }^{2} .{ }^{1}$ rheumatology,

${ }^{2}$ immunology, Institute of Cardiology, Kyiv, Ukraine

Background: To compare bone metabolism in pre- and postmenopausal women with RA

Objectives: GCs and inflammation lead to BMD loss through increase the expression of RANK-ligand and decrease the expression of osteoprotegerin in stromal and osteoblastic cells. Determination of indicators relevant for clinical practice for identification a high-risk BMD loss group of RA patients is needed.

Methods: The bone turnover markers were analyzed in 58 women with RA: 36 premenopausal (PreM) and 22 postmenopausal (PM). The two groups were significantly different in age $(35.9 \pm 8.7$ PreM vs $57.1 \pm 4.1 \mathrm{PM})$ and $\mathrm{BMI}(23.8 \pm 4.3$ PreM vs 28.6 \pm 5.0 PM). By disease duration (8.9 \pm 5.7 years vs $10.0 \pm 6.1$ years) disease activity by DAS28, x-ray changes, GC, MTX and biologic therapy both group were comparable. BMD was measured in 3 part of the skeleton: hip, lumbar spine, distal part of forearm. Serum levels of $25(\mathrm{OH})$ vitamin $D(V i t)$ ), parathormone (PTH), osteoprotegerin (OPG), RANKL were analyzed.

Results: Low level of Vit D $(25(\mathrm{OH}) \mathrm{D}<30 \mathrm{ng} / \mathrm{ml})$ was observed in $46.6 \%$ of patient and was preferable in PreM women. Vit $D$ deficiency was associated with age $(R=-0.31, p<0.05)$ and positive anti-CCP $(R=-0.46, p<0.05)$. The mean level of PTH was normal in $74.1 \%$. Serum OPG level was significantly higher in more than $50 \%$ of patients. It was associated with presence of erosion ( $R=0.97$ $\mathrm{p}<0.05)$, decreased cortical index $(R=-0.28, p<0.05)$ in PreM patient and DAS28 $(R=-0.44, p<0.05)$ in all patients. High level of RANKL was preferable in PM women $(47.6 \%)$, decreased level more often was observed in PreM patient (38.9\%). In PM patients increased level of RANKL was correlated with BMI, duration of menopausa and DAS28, in PreM - only with DAS28. In PreM women the changes in serum levels of PTH and vitamin D was negatively correlated with levels of RANKL and C-TP, low level of vitamin D had negative correlation with OPG ( $R$ - 0.47$)$. According to lineal regression analysis positive correlation between disease duration, Sharp van der Heijde erosion score and PTH $(r=0.31 ; p=0.01$, $r 2=0.11$ ) was observed in all patients, more often in PreM women. Low spine BMD in PreM associated with high PTH $(R=0.82, p<0.05)$, in $\mathrm{PM}-$ with high $\mathrm{PTH}$, OPG/RANKL and low level of RANKL. Low hip neck BMD correlated with decreased level of vit D in PM patients. Low levels of vit D, C-TP and high levels of OPG and OPG/RANKL index was significantly associated with forearm low BMD in all patients. In PreM women strong association between low vit $D(R=-0.60$, $\mathrm{p}<0.05)$, high OPG $(R=0.89, p<0.05)$ and $B M D$ in medium part of forearm was observed.

Conclusions: In premenopausal women vitamin $\mathrm{D}$ deficiency had high predictive value for decrease BMD in the medium part of forearm, high level of PTH - for decrease BMD in the spine. In PreM RA patients low Vit D, low cortical index and high PTH can be considered as an indication for BMD assessment. PM women with high BMI, longstanding postmenopausal period and high disease activity may be candidate for bone markers assessment.

\section{REFERENCES:}

[1] Schett G, Kiechl S, Weger S, et al. High-sensitivity C-reactive protein and risk of non-traumatic fractures in the Bruneck study. Arch Intern Med 2006;166:2495-501.

[2] Charactcharoenwitthaya N, Khosla S, Atkinson EJ, et al. Effect of blockade of TNF- $\alpha$ and interleukine- 1 action on bone resorption in early postmenopausal women. J Bone Miner Res 2007;22:724-9.

Disclosure of Interest: None declared

DOI: 10.1136/annrheumdis-2018-eular.5272 